\title{
Intra-articular Lesions and Clinical Outcomes in Traumatic Anterior Shoulder Dislocation Associated with Greater Tuberosity Fracture of the Humerus
}

\author{
Kuk Pil Lim, In Seung Lee, In-Bo Kim ${ }^{\square}$ \\ Department of Orthopedic Surgery, Busan Bumin Hospital, Busan, Korea
}

Background: This study investigated and evaluated the clinical outcomes of intra-articular lesions of traumatic anterior shoulder dislocation (TASD) associated with greater tuberosity (GT) fracture of the humerus.

Methods: Subjects included 20 patients who were surgically or non-surgically treated for GT fracture of the humeurs with TASD, and followed-up for at least 2 years. The mean follow-up period was 54.1 months (range, 24-105 months). Of the 20 patients, 12 were treated surgically. Intra-articular lesions were identified randomly on magnetic resonance imaging scans (repeated thrice) by experienced radiologists and orthopedic surgeons. The accompanying intra-articular lesions were left untreated. Clinical outcomes were evaluated by Simple Shoulder Test (SST) and Western Ontario Shoulder Instability index (WOSI) at the last follow-up.

Results: Intra-articular lesions were identified in 19 patients: 7 Bankart lesions, 15 humeral avulsion of the glenohumeral ligament lesions, 3 glenoid avulsion of the glenohumeral ligament lesion, and 6 inferior capsular tears. Two or more intra-articular lesions were identified in 6 patients. The mean SST score was 10.9 and the mean WOSI score was 449.3 at the last follow-up.

Conclusions: For GT fracture of the humerus with TASD, a high frequency of diverse intra-articular lesions was identified. There were no incidence of recurrent shoulder dislocations, and good clinical outcomes were obtained without treatment of the intra-articular lesions. We thereby comprehend that although intra-articular lesions may occur in TASD associated with GT fracture of the humeurs, merely treating the GT fracture of the humerus is sufficient.

(Clin Shoulder Elbow 2017;20(4):195-200)

Key Words: Intra-articular lesions; Traumatic anterior shoulder dislocation; Greater tuberosity fracture

\section{Introduction}

Isolated fractures of the greater tuberosity (GT) constitue $17 \%$ to $21 \%$ of proximal humerus fractures, and $15 \%$ to $30 \%$ of them are associated with traumatic anterior shoulder dislocation (TASD). ${ }^{1,2)}$ When the dislocated shoulder associated with GT fracture was reducted, most of the GT fracture of humerus were reducted simultaneously and conservative treatment was performed. ${ }^{3)}$ Nonsurgical treatment was the mainstay for minimally displaced GT fractures (less than $10 \mathrm{~mm}$ of displacement). ${ }^{4,5)}$ Recent cadaveric and clinical studies have indicated that $5 \mathrm{~mm}$ of displacement can result in a poor functional outcome. ${ }^{6-9)}$ Bigliani and Flatow ${ }^{10)}$ suggested that surgical treatment is necessary when displacement of the GT exceeds $5 \mathrm{~mm}$. With displacement, the altered function of the rotator cuff tendons or direct mechanical block can lead to loss of shoulder abduction and strength. ${ }^{4,5,11)}$ Hence, surgical treatment is necessary in few cases where displacement of the GT was more than $5 \mathrm{~mm}$ and angulation was more than 45 degrees. ${ }^{10)}$ Hence, in our study, when displacement of GT exceeded $5 \mathrm{~mm}$, surgical treatment was performed. When the GT fracture of the humeurs had accompanying TASD, it was common to treat only for the fracture. However, regardless

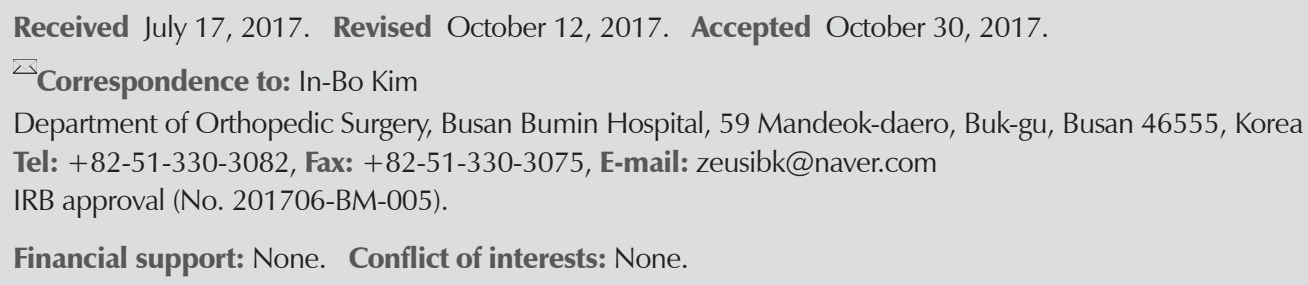


of shoulder dislocation, there is a high incidence of soft tissue injuries in the GT fracture of the humerus; when these findings are overlooked, prognosis is poor and limitation of movement may occur. ${ }^{12)}$ Conversely, it has been reported that conservative treatment of intra-articular lesions alone yield good clinical outcomes. ${ }^{13)}$ In the GT fracture of the humerus with TASD, there are differing opinions regarding the treatment of intra-articular lesions. This study therefore undertook to investigate the intraarticular lesions of TASD associated with GT fracture of the humerus, and evaluate the clinical outcomes after treating the GT fracture alone.

\section{Methods}

From 2010 to 2015, 24 patients who visited emergency room had GT fracture with associated TASD. Four patients were excluded since their magnetic resonance imaging (MRI) scan was not performed; the remaining 20 patients included in the study were surgically or non-surgically treated for GT fracture of the humerus with TASD, and followed-up for at least 2 years. Dislocation of the shoulder was reducted first in the emergency room. The mean follow-up period was 54.1 months (range, 24-105 months). The mean patient age was 54.6 years (range, 29-84 years); for non-surgical treatment: 50.0 years (range, 29-54 years), for surgical treatment: 57.7 years (range, 36-77 years). The different causes of injury included car accidents (6 cases), falling down (2 cases) and slipping (12 cases) (Table 1, 2). Surgical interventions for GT fracture in 12 of the 20 patients included open reduction and internal fixation (ORIF) with cannulated screw (3 cases), ORIF with plate (3 cases), and arthroscopic reduction and fixation with the suture bridge technique $(6$ cases). There was no specific indication for the fixation method employed, which was selected according to the bone fragment size, bone density and degree of comminution. The remaining 8

Table 1. Patient Data

\begin{tabular}{|c|c|c|c|c|c|c|c|c|}
\hline $\begin{array}{c}\text { Patient } \\
\text { No. }\end{array}$ & Sex & Age (yr) & Cause & Accompanying lesion & Treatment & $\begin{array}{l}\text { Follow-up } \\
(\mathrm{mo})\end{array}$ & $\mathrm{SST}^{*}$ score & WOSI $^{\dagger}$ score \\
\hline \multicolumn{9}{|c|}{ Non-surgical } \\
\hline 1 & $\mathrm{~F}$ & 29 & $\mathrm{TA}$ & HAGL, Inf. capsule tear & $\mathrm{CR}$ & 40 & 12 & 375 \\
\hline 2 & M & 40 & $\mathrm{TA}$ & Bankart lesion, Inf. capsule tear & $\mathrm{CR}$ & 99 & 12 & 435 \\
\hline 3 & M & 47 & TA & Inf. capsule tear & $\mathrm{CR}$ & 98 & 12 & 355 \\
\hline 4 & $\mathrm{~F}$ & 61 & Fell down & HAGL & CR & 66 & 10 & 470 \\
\hline 5 & $\mathrm{~F}$ & 36 & Slipped & HAGL & CR & 28 & 12 & 415 \\
\hline 6 & $\mathrm{M}$ & 58 & Slipped & Bankart lesion, GAGL, Inf. capsule tear & CR & 78 & 12 & 485 \\
\hline 7 & $\mathrm{~F}$ & 84 & Slipped & HAGL, RCT (partial) & $\mathrm{CR}$ & 27 & 7 & 600 \\
\hline 8 & $\mathrm{~F}$ & 45 & Slipped & HAGL & $\mathrm{CR}$ & 24 & 12 & 445 \\
\hline \multicolumn{9}{|l|}{ Surgical } \\
\hline 9 & $\mathrm{~F}$ & 49 & $\mathrm{TA}$ & Bankart lesion & Cannulated screw & 104 & 12 & 440 \\
\hline 10 & $\mathrm{~F}$ & 54 & $\mathrm{TA}$ & HAGL & Suture bridge & 105 & 12 & 390 \\
\hline 11 & $\mathrm{M}$ & 66 & $\mathrm{TA}$ & Bankart lesion, HAGL & Suture bridge & 51 & 8 & 460 \\
\hline 12 & M & 53 & Fell down & HAGL & Plate & 25 & 12 & 405 \\
\hline 13 & $\mathrm{M}$ & 36 & Slipped & Inf. capsule tear & Cannulated screw & 57 & 12 & 410 \\
\hline 14 & $\mathrm{~F}$ & 46 & Slipped & Bankart lesion, HAGL, RCT (partial) & Bankart repair, suture bridge & 53 & 12 & 500 \\
\hline 15 & M & 55 & Slipped & HAGL, Bankart lesion & Bankart repair, suture bridge & 30 & 12 & 480 \\
\hline 16 & $\mathrm{~F}$ & 58 & Slipped & Bankart lesion, HAGL & Plate & 26 & 11 & 490 \\
\hline 17 & M & 59 & Slipped & Inf. capsule tear, RCT (full) & RCT repair, suture bridge & 43 & 8 & 500 \\
\hline 18 & $\mathrm{~F}$ & 69 & Slipped & None & Plate & 28 & 10 & 390 \\
\hline 19 & $\mathrm{~F}$ & 70 & Slipped & HAGL & Suture bridge & 52 & 10 & 435 \\
\hline 20 & $\mathrm{~F}$ & 77 & Slipped & HAGL & Cannulated screw & 47 & 9 & 475 \\
\hline Total & & 54.6 & & & & 54.1 & 10.9 & 449.3 \\
\hline
\end{tabular}

SST: Simple Shoulder Test, WOSI: Western Ontario Shoulder Instability index, F: female, M: male, TA: traffic accident, HAGL: humeral avulsion of the glenohumeral ligament, Inf: inferior, GAGL: glenoid avulsion of the glenohumeral ligament, RCT: rotator cuff tear, CR: close reduction.

${ }^{*}$ The scores range from 0 (worst) to 12 (best). ${ }^{\dagger}$ The scores range from 0 (best) to 2,100 (worst). 
patients were treated non-surgically using shoulder immobilization with K-sling brace for 2 weeks. After 2 weeks, the patients sequentially underwent passive exercise, active assistive exercise, active exercise and strengthening exercise. In all cases, MRI scan was performed after the dislocated shoulder was reducted, and the presence of accompanying injury lesions was confirmed. Intra-articular lesions were randomly identified in the MRI scans, which were repeated and evaluated three times by experienced radiologists and orthopedic surgeons. Intra-articular lesions were additionally identified by shoulder arthroscopy, in cases where performed. This study was approved by the institutional review board.

Table 2. Patient Data

\begin{tabular}{|cc|}
\hline \multicolumn{1}{|c}{ Parameter } & Value \\
\hline No. of patient & 20 \\
\hline Age (yr) & $54.6(29-84)$ \\
\hline Sex & 8 \\
\hline Male & 12 \\
\hline Female & \\
\hline Cause & 6 \\
\hline Car accident & 2 \\
\hline Fell down & 12 \\
\hline Slipped & $54.1(24-105)$ \\
\hline Follow-up period (mo) & \\
\hline
\end{tabular}

Values are presented as number only or median (range).

\section{Measures of Clinical Outcomes}

At the last follow-up, clinical outcomes were evaluated by the Simple Shoulder Test (SST) and the Western Ontario Shoulder Instability index (WOSI). The SST consists of 12 questions with dichotomous (yes/no) response options. For each question, the patient indicates whether they are able or not to do the activity. The scores range from 0 (worst) to 12 (best). ${ }^{14)}$ The WOSI is a visual analogue score made up of 21 questions subdivided into four domains: physical symptoms, sports/recreation/work, lifestyle, and emotions. The scores range from 0 (best) to 2,100 (worst); a lower score indicates a better quality of life and good clinical outcomes. ${ }^{15)}$ Mann-Whitney U-test was performed to assess the differences of SST and WOSI between surgically treated and non-surgically treated groups. The IBM SPSS software package ver. 20.0 (IBM Co., Armonk, NY, USA) was used for statistical analysis, with $p$-value set at 0.05 .

\section{Results}

The intra-articular lesions were identified in 19 patients; these included 7 Bankart lesions (Fig. 1), 15 humeral avulsion of the glenohumeral ligament (HAGL) lesions, 3 glenoid avulsion of the glenohumeral ligament (GAGL) lesions, and 6 inferior capsular tears (Table 1, 3). Two or more intra-articular lesions were identified in 6 patients: HAGL and inferior capsular tear (4 patients), HAGL and Bankart lesion (4 patients), and Bankart lesion and inferior capsular tear (1 patient). One patient had three lesions, namely, GAGL, Bankart lesion and inferior capsular tear. Other accompanying lesions were rotator cuff tears (1 complete and 2 partial supraspinatus tear). No significant differences were
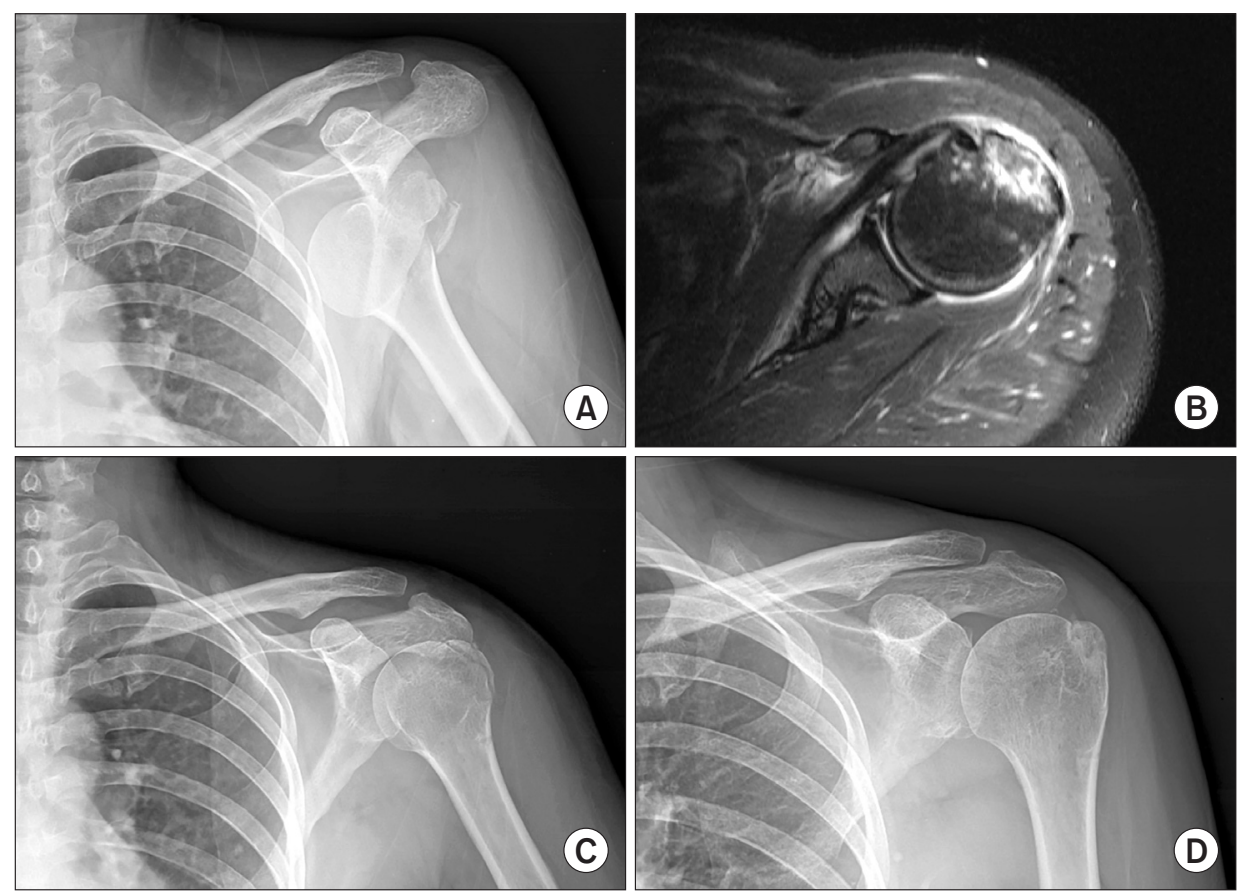

Fig. 1. (A) Right shoulder anterior dislocation caused by slipping (case 14). (B) Bankart lesion on magnetic resonance imaging scan. (C) Postoperative radiography. (D) Last follow-up radiography. 
Table 3. Accompanying Lesion

\begin{tabular}{lc}
\hline \multicolumn{1}{c}{ Variable } & Number \\
\hline Bankart lesion & 7 \\
Capsular tear & 6 \\
\hline Inferior capsular tear & \\
Avulsion of the inferior glenohumeral ligament & 15 \\
HAGL & 3 \\
GAGL & \\
\hline Rotator cuff tear & 1 \\
\hline Complete rotator cuff tear & 2 \\
\hline Partial rotator cuff tear & 34 \\
\hline Total
\end{tabular}

HAGL: humeral avulsion of the glenohumeral ligament, GAGL: glenoid avulsion of the glenohumeral ligament.

observed in the types and frequency of intra-articular lesions in surgically and non-surgically treated patients.

\section{Clinical Outcome}

The SST and WOSI scores were evaluated at the last followup. The mean SST score was 10.9 in all patients. The difference in the SST scores for conservative treatment $(11.1 \pm 1.8)$ and surgical treatment $(10.7 \pm 1.6)$ was statistically not significant $(p=0.473)$. The mean WOSI score was assessed at 449.3 , with no statistical significance between the conservative treatment $(447.5 \pm 75.8)$ and surgical treatment $(450.4 \pm 42.0)(p=0.624)$ (Table 4,5$)$. No recurrence of shoulder subluxation or dislocation was observed during follow-up. Also, there were no occurrences of any other complications, such as vascular injury, stiffness and non-union.

\section{Discussion}

The GT of humerus is an important component of the subacromial gliding mechanism, and serves as the insertion for the rotator cuff tendons which are the dynamic stabilizers and controllers of the shoulder. ${ }^{3)}$ Hence, improper management of a GT fracture of humerus associated with a TASD results in impaired shoulder function. ${ }^{4,5,11)}$ Advances in surgical techniques and instruments have resulted in a remarkable development in the treatment of acute GT fracture of humerus with TASD in recent years. Arciero et al. ${ }^{16)}$ and Barber et al. ${ }^{17)}$ reported that early surgical treatment reduces the recurrence and prevents additional bone or soft tissue damage, thereby avoiding secondary damage. However, although there are many studies on the treatment of GT fracture of humerus with TASD, little has been reported on the intra-articular lesions and their impact on clinical outcomes. The GT fractures associated with glenohumeral dislocation show
Table 4. Clinical Score of Total ${ }^{\star}$

\begin{tabular}{lc}
\hline \multicolumn{1}{c}{ Parameter } & Value \\
\hline Age $(\mathrm{yr})$ & $54.6 \pm 14.2$ \\
\hline SST & $10.9 \pm 1.7$ \\
\hline WOSI score $(2,100)$ & $449.3 \pm 56.0$ \\
\hline Physical symptoms (1,000) & $191.5 \pm 14.9$ \\
\hline Sports/recreation/work (400) & $102.0 \pm 20.4$ \\
\hline Lifestyle (400) & $93.5 \pm 16.1$ \\
\hline Emotion (300) & $62.3 \pm 12.3$ \\
\hline Follow-up period (mo) & $54.1 \pm 31.8$ \\
\hline
\end{tabular}

Values are presented as mean \pm standard deviation.

SST: Simple Shoulder Test, WOSI: Western Ontario Shoulder Instability index. ${ }^{\star}$ Total $(\mathrm{n}=20)$.

Table 5. Clinical Score of Individual

\begin{tabular}{lccc}
\hline \multicolumn{1}{c}{ Variable } & $\begin{array}{c}\text { Non-operation } \\
(\mathrm{n}=8)\end{array}$ & $\begin{array}{c}\text { Operation } \\
(\mathrm{n}=12)\end{array}$ & $p$-value \\
\hline Age $(\mathrm{yr})$ & $50.0 \pm 17.4$ & $57.7 \pm 11.5$ & 0.208 \\
\hline SST & $11.1 \pm 1.8$ & $10.7 \pm 1.6$ & 0.473 \\
\hline WOSI score (2,100) & $447.5 \pm 75.8$ & $450.4 \pm 42.0$ & 0.624 \\
\hline Physical symptoms (1,000) & $195.0 \pm 19.3$ & $189.2 \pm 11.4$ & 0.624 \\
\hline Sports/recreation/work (400) & $100.0 \pm 21.0$ & $103.3 \pm 20.7$ & 0.571 \\
\hline Lifestyle (400) & $90.6 \pm 20.9$ & $95.4 \pm 12.5$ & 0.305 \\
\hline Emotion (300) & $61.9 \pm 19.3$ & $62.5 \pm 5.0$ & 0.792 \\
\hline Follow-up period (mo) & $53.0 \pm 37.0$ & $49.8 \pm 29.4$ & 0.910 \\
\hline
\end{tabular}

Values are presented as mean \pm standard deviation.

SST: Simple Shoulder Test, WOSI: Western Ontario Shoulder Instability index.

a much higher frequency of labral lesions than those with verified nondislocation. ${ }^{18)}$ Kim and $\mathrm{Ko}^{19)}$ reported on arthroscopic findings that the Bankart lesion, which results in shoulder instability, frequently occurs in anterior shoulder dislocations than in the GT fracture of humerus with TASD. Also, differences were observed in intra-articular lesions between the GT fracture of the humerus with TASD and in isolated acute shoulder dislocation. In the GT fracture of humerus with TASD, there is a lesser occurrence of typical Bankart lesions. A higher frequency of Perthes lesion which does not require arthroscopic repair, glenolabral articular disruption lesion, free anterior labral periosteal sleeve avulsion and capsular tear was observed. ${ }^{3)}$ In this study, two Bankart lesions with severe displacement and one complete rotator cuff tear (supraspinatus tendon tear) were treated by arthroscopic repair, whereas other intra-articular lesions were treated conservatively. Bottoni et al. ${ }^{20)}$ suggested that capsular tear less than $1 \mathrm{~cm}$ in acute shoulder dislocation could be healed naturally with good clinical outcomes. Kim et al. ${ }^{3)}$ reported that conservative treatment of intra-articular lesions, except Bankart lesion and 
bony Bankart lesion, may also yield good clinical results. In our study, except Bankart lesion and rotator cuff tear, the conservative treatment of intra-articular lesions yielded satisfactory clinical outcomes. Treatment of merely the fracture in the GT fracture of humerus with TASD was also successful in general. Except for the bony Bankart lesion, all accompanying intra-articular lesions could be confirmed by simple radiograph and computed tomography scans, and were normally healed and did not rupture. Therefore, accompanying intra-articular lesions generally found in the GT fracture of humerus with TASD obtained good clinical outcomes by conservative treatment alone. Reeves ${ }^{21)}$ reported that in the anterior capsular structures of the shoulder, the labrum was weaker in younger people, and the articular capsule and subscapularis tendon was weaker in the elderly. Since there was no set age limit in our study, the possibility of a previous intra-articular lesion in the shoulder was not considered, and we were unable to accurately assess the effect of intra-articular lesions according to age in the GT fracture of humerus with TASD; hence, further studies are required. All intra-articular lesions were identified by MRI scans without shoulder arthroscopy; hence, confirmation of intra-articular lesions was not accurate. In conservative treatment, the intra-articular lesions were identified by MRI scan but not by shoulder arthroscopy. Furthermore, the intra- articular lesion healing was not confirmed by a second MRI scan after enough time had elapsed for healing. In 3 patients who underwent arthroscopic surgery, the intra-articular lesions were additionally identified by the shoulder arthroscopy.

\section{Conclusion}

For GT fracture of humerus with TASD, various intra-articular lesions were identified at a high occurrence. There was no incident of recurrent shoulder dislocation, and good clinical outcomes were obtained without treatment of intra-articular lesions. Our results indicate that in cases of TASD with the GT fracture of humerus having associated intra-articular lesions, treatment of only the GT fracture of humerus could be performed with effective clinical consequences.

\section{References}

1. Lind T, Krøner K, Jensen J. The epidemiology of fractures of the proximal humerus. Arch Orthop Trauma Surg. 1989; 108(5):285-7.

2. Platzer P, Kutscha-Lissberg F, Lehr S, Vecsei V, Gaebler C. The influence of displacement on shoulder function in patients with minimally displaced fractures of the greater tuberosity. Injury. 2005;36(10):1185-9.

3. Kim DS, Yoon YS, Lee DK, Park HK, Park JH, Shin J. Accompanying lesions and clinical results in the greater tuberosity fracture of the humerus with anterior shoulder dislocation under the age of forty. Clin Shoulder Elbow. 2011;14(1):20-6.

4. Neer CS. Displaced proximal humeral fractures. I. Classification and evaluation. J Bone Joint Surg Am. 1970;52(6):107789.

5. Mclaughlin HL. Dislocation of the shoulder with tuberosity fracture. Surg Clin North Am. 1963;43(6):1615-20.

6. Bigliani LU, Flatow EL, Pollock RG. Fractures of the proximal humerus. In: Harryman DT, Matsen FA, Rockwood CA, et al, eds. The shoulder: Vol. 1. 2nd ed. Philadelphia (PA): WB Saunders; 1998. 337-90.

7. Bono CM, Renard R, Levine RG, Levy AS. Effect of displacement of fractures of the greater tuberosity on the mechanics of the shoulder. J Bone Joint Surg Br. 2001;83(7):1056-62.

8. lannotti J, Sidor M. Malunions of the proximal humerus. In: Warner J, lannotti J, Gerber C, eds. Complex and revision problems in shoulder surgery. Philadelphia (PA): LippincottRaven; 1997. 245-64.

9. Craig E. Open reduction and internal fixation of greater tuberosity fractures, malunions and nonunions. In: Craig E, ed. Master techniques in orthopaedic surgery: the shoulder. New York (NY): Raven Press; 1995. 289-307.

10. Bigliani L, Flatow E. The shoulder. 3rd ed. Philadelphia (PA): WB Saunders; 1998. 337-89.

11. Neviaser JS. Complicated fractures and dislocations about the shoulder joint. J Bone Joint Surg Am. 1962;44(5):984-8.

12. Park TS, Choi IY, Kim YH, Park MR, Shon JH, Kim SI. A new suggestion for the treatment of minimally displaced fractures of the greater tuberosity of the proximal humerus. Bull Hosp Jt Dis. 1997; 56(3):171-6.

13. Williams GR Jr, Wong KL. Two-part and three-part fractures: open reduction and internal fixation versus closed reduction and percutaneous pinning. Orthop Clin North Am. 2000;31(1):1-21.

14. Lippitt SB, Harryman DT, Matsen FA. A practical tool for evaluation of function: the simple shoulder test. In: Matsen FA, Fu FH, Hawkins RJ, eds. The shoulder: a balance of mobility and stability. Rosemont (IL): American Academy of Orthopaedic Surgery; 1993. 501-18.

15. Kirkley A, Griffin S, McLintock H, Ng L. The development and evaluation of a disease-specific quality of life measurement tool for shoulder instability. The Western Ontario Shoulder Instability Index (WOSI). Am J Sports Med. 1998;26(6):764-72.

16. Arciero RA, Wheeler JH, Ryan JB, McBride JT. Arthroscopic Bankart repair versus nonoperative treatment for acute, initial anterior shoulder dislocations. Am J Sports Med. 1994;22(5):589-94.

17. Barber FA, Ryu RK, Tauro JC. Should first time anterior shoulder dislocations be surgically stabilized? Arthroscopy. 2003;19(3):305-9.

18. Schai PA, Hintermann B, Koris MJ. Preoperative arthroscopic assessment of fractures about the shoulder. Arthroscopy. 
1999;15(8):827-35.

19. Kim YK, Ko KC. Arthroscopic findings in acute shoulder dislocation associated with a fracture of greater tuberosity of the humerus. J Korean Orthop Assoc. 2000;35(3):437-42.

20. Bottoni CR, Wilckens JH, DeBerardino TM, et al. A prospective, randomized evaluation of arthroscopic stabilization versus nonoperative treatment in patients with acute, traumatic, firsttime shoulder dislocations. Am J Sports Med. 2002;30(4):57680.

21. Reeves B. Experiments on the tensile strength of the anterior capsular structures of the shoulder in man. J Bone Joint Surg Br. 1968;50(4):858-65. 